\title{
Bifurcation and stability analysis of a two step model for monitoring anaerobic digestion processes ${ }^{\text {th }}$
}

\author{
B. Benyahia ${ }^{\mathrm{a}, \mathrm{b}, *}$, T. Sari ${ }^{\mathrm{b}, \mathrm{c}}$, B. Cherki ${ }^{\mathrm{a}}$, J. Harmand ${ }^{\mathrm{b}, \mathrm{d}}$ \\ ${ }^{a}$ Université de Tlemcen, Laboratoire d'Automatique, BP 119 Tlemcen 13000, Algérie \\ ${ }^{b}$ Projet Modemic, Inra/Inria, UMR Mistea, 2 place Pierre Viala 34060 Montpellier, \\ France \\ ${ }^{c}$ Irstea, UMR Itap, 361 rue Jean-Franois Breton, 34196 Montpellier, France \\ ${ }^{d}$ INRA, UR050, Laboratoire de Biotechnologie de l'Environnement, Avenue des Etangs, \\ Narbonne F-11100, France
}

\begin{abstract}
This paper deals with the equilibria and stability analysis of the two step anaerobic model initially proposed by [12] to describe the dynamical behavior of an anaerobic fixed-bed wastewater treatment process. In a first part, the model is analyzed: its equilibria and their stability are established considering qualitative properties of the kinetics. In a second part, it is shown that the overloading tolerance (denoted herein OT), a parameter proposed in [9] to monitor anaerobic processes on-line, may not be suitable for monitoring the system and even causes serious problems under certain functioning conditions. Based on the analysis results established in the first part, a modified OT is proposed and evaluated in simulation.
\end{abstract}

Keywords: Anaerobic digestion, biotechnology, steady state analysis,

\footnotetext{
This work was supported by AiresSud (project 7015), Coadvise (project FP7PEOPLE-IRSES-2008 No. 230833) and Treasure (an Inria Euromed 3+3 project, http: //www.treasure.fr). This article is an extension of the CAB 2010 proceeding [2]. *Corresponding author. Tel.: +33(0)4 996124 22; fax: +33(0)4 67521427

Email address: benyahia@supagro.inra.fr (B. Benyahia)
} 
equilibrium, model approximation, risk analysis

\section{Introduction}

Depending on the objective for which they have been developed, anaerobic digestion models can be classified in two main groups. On one hand, there are high dimensional models developed by practioners to capture accurately the phenomenologic behavior of anaerobic digestion systems following the idea that all the available information and knowledge should be included in the models. The famous ADM1 model typically falls into this class of models, cf. ([7]). On the other hand, simplified models are developed for monitoring and control design purposes by control engineers for optimizing the functioning of bioprocesses. If it is quite obvious that a deep mathematical analysis of complex models is very difficult to tackle, for not saying impossible. As surprising as it may appear the analysis of simple models such as the two step anaerobic model proposed by [12] (hereafter denoted as the AM2 model) has never been realized in a generic way. Two steps models have been nonetheless widely used for on-line monitoring anaerobic processes because of both its relative simplicity and its high capacity to reproduce the dynamical behaviour of the main operational parameters of the process. To derive specific monitoring and control strategies, a number of authors performed more or less deep analyses of simple anaerobic models.

S. Shen and G.C. Premier [14] analyzed the stability of equilibria and presented a bifurcation analysis of an anaerobic digestion model according to some operating parameters. They derived from a six dimensional model [15] a two dimensional model including only the methanogenesis step by 
reducing and/or eliminating the stable and rapid dynamics.

To study the AM2 model, J. Hess and O. Bernard [9] proposed to use a reduced model in considering that what enters the second step of the process (the methanogenesis step) is what comes "in the worst case" from the acidogenesis process. Using their results, they proposed a monitoring strategy based on a stability criteria named "overloading tolerance" in monitoring a "destabilization risk index", cf. [8].

M. Sbarciog et al [10] proposed a methodology to estimate the separatrix between the stable attraction basins of the equilibria. However, their analysis was based on specific kinetics. In addition, it was not realized in the original coordinates and their results are thus quite a bit difficult to interpret.

N. Dimitrova and M. Krastanov [11] studied the equilibria stability and performed a bifurcation analysis of the AM2 model according to the dilution rate (control variable). However, they restricted their attention to specific kinetics and conditions depending on the influent substrate concentration.

Recently, I. Simenov and S. Diop [6] analyzed the local stability of some anaerobic digestion models. They initially studied a two dimensional simple model restricting their attention to the methanogenic step, by considering specific kinetics (Monod, Contois or Haldane). Furthermore, they also considered a four dimensional anaerobic digestion model (including acidogenesis and methanogenesis). However, in this last case, they restricted their attention to Monod kinetics excluding the inhibition by the Volatile Fatty Acids.

To summarize the state on the art about the mathematical analysis of two step models of anaerobic systems, one may say that all available studies used specific kinetics and authors restricted their attention to a limited number 
of functioning conditions.

In the present paper, we propose a mathematical analysis of equilibria and their stability of a two steps model (AM2). The proposed analysis can be said to be "generic" in the sense we do not specify kinetics but rather define qualitative hypotheses on which we base our results. It should be noticed that this genericity provides the possibility of extending our results to the analysis of any 2 step bioprocesses where the second step can be inhibited by its own substrate. In addition, we revisit the "overloading tolerance" parameter (denoted OT herein) within this general analysis framework in order to be able to monitor anaerobic processes under more general functioning conditions that those initially considered in [9] that are not valid in the case of equilibria bistability.

The paper is organized as follows. First, the AM2 model is recalled. Depending on the model and input parameters, equilibria are characterized and their stability is analyzed. Then, the overloading parameter initially proposed by [9] is revisited and a modified version is proposed at the light of the previous performed mathematical analysis. Then, we illustrate and discuss our results in simulation before conclusions and perspectives are drawn.

\section{Mathematical model}

We consider the mathematical model of the anaerobic process based on 2 main reactions, where substrate $S_{1}$ is degraded into substrate $S_{2}$ by bacteria $X_{1}$ and then $S_{2}$ is degraded by bacteria $X_{2}$. This model, initially proposed by [12] and partially analyzed in [9] is given by:

$$
\dot{S}_{1}=D\left(S_{1 i n}-S_{1}\right)-k_{1} \mu_{1}\left(S_{1}\right) X_{1},
$$




$$
\begin{aligned}
\dot{X}_{1} & =\left(\mu_{1}\left(S_{1}\right)-\alpha D\right) X_{1}, \\
\dot{S}_{2} & =D\left(S_{2 i n}-S_{2}\right)+k_{2} \mu_{1}\left(S_{1}\right) X_{1}-k_{3} \mu_{2}\left(S_{2}\right) X_{2}, \\
\dot{X}_{2} & =\left(\mu_{2}\left(S_{2}\right)-\alpha D\right) X_{2},
\end{aligned}
$$

where $D$ is the dilution rate, while $S_{1 \text { in }}$ and $S_{2 \text { in }}$ are the input substrate concentrations respectively. Parameters $k_{i}$ are pseudo-stochiometric coefficients associated to the bioreactions. $\alpha \in[0,1]$ is a parameter allowing us to decouple the HRT (Hydraulic Retention Time) and the SRT (Solid Retention Time). The kinetics $\mu_{1}$ and $\mu_{2}$ are of Monod and Haldane type, respectively. However, in the sequel, we will consider generic kinetics $\mu_{1}$ and $\mu_{2}$, satisfying the following qualitative properties:

Hypothesis 1. $\mu_{1}\left(S_{1}\right)$ is an increasing function for $S_{1} \geq 0$, with $\mu_{1}(0)=0$ and $\mu_{1}(+\infty)=m_{1}$. We assume that the derivative $\mu_{1}^{\prime}\left(S_{1}\right)$ of $\mu_{1}\left(S_{1}\right)$ with respect to $S_{1}$ satisfies the property : $\mu_{1}^{\prime}\left(S_{1}\right)>0$ for all $S_{1} \geq 0$.

Hypothesis 2. $\mu_{2}\left(S_{2}\right)$ is an increasing function for $0 \leq S_{2}<S_{2}^{M}$, has a maximum $\mu_{2}\left(S_{2}^{M}\right)>0$ for $S_{2}=S_{2}^{M}$ and then decreasing for $S_{2}>S_{2}^{M}$, with $\mu_{2}(0)=0$ and $\mu_{2}(+\infty)=0$. We assume that the derivative $\mu_{2}^{\prime}\left(S_{2}\right)$ of $\mu_{2}\left(S_{2}\right)$ with respect to $S_{2}$ satisfies the property : $\mu_{2}^{\prime}\left(S_{2}\right)>0$ for $0 \leq S_{2}<S_{2}^{M}$, and $\mu_{2}^{\prime}\left(S_{2}\right)<0$ for $S_{2}>S_{2}^{M}$.

In [11], one considered model (1-4) in the case when $\mu_{1}$ is a Monod kinetics, $\mu_{2}$ is a Haldane kinetics and $S_{2 i n} \geq S_{2}^{M}$. They studied the equilibria of the system and their local one-parameter bifurcations (according to the dilution rate). Here we consider the general case of $\mu_{1}$ and $\mu_{2}$ satisfying Hypotheses 1 and 2 and without the restriction $S_{2 i n} \geq S_{2}^{M}$. 


\subsection{Equilibria of model}

We use the following notations. Let $\alpha D<m_{1}$, we denote:

$$
S_{1}^{*}(D)=\mu_{1}^{-1}(\alpha D)
$$

the root of equation $\mu_{1}\left(S_{1}\right)=\alpha D$.

If $\alpha D \geq m_{1}$, this equation has no solution and we let $S_{1}^{*}(D)=+\infty$.

If $S_{1 i n} \geq S_{1}^{*}(D)$ we denote:

$$
S_{2 i n}^{*}(D)=S_{2 i n}+\frac{k_{2}}{k_{1}}\left(S_{1 i n}-S_{1}^{*}(D)\right), \quad X_{1}^{*}(D)=\frac{1}{k_{1} \alpha}\left(S_{1 i n}-S_{1}^{*}(D)\right) .
$$

Let $\alpha D<\mu_{2}^{M}:=\mu_{2}\left(S_{2}^{M}\right)$. We denote $S_{2}^{1 *}(D)<S_{2}^{2 *}(D)$ the roots of equation $\mu_{2}\left(S_{2}\right)=\alpha D$.

If $\alpha D=\mu_{2}^{M}$, this equation has only one solution and we let $S_{2}^{1 *}(D)=S_{2}^{2 *}(D)$.

If $\alpha D>\mu_{2}^{M}$, this equation has no solution and we let $S_{2}^{1 *}(D)=+\infty$.

If $S_{2 i n} \geq S_{2}^{i *}(D)$ we denote:

$$
X_{2}^{i}(D)=\frac{1}{k_{3} \alpha}\left(S_{2 i n}-S_{2}^{i *}(D)\right), \quad i=1,2 .
$$

If $S_{1 i n} \geq S_{1}^{*}(D)$ and $S_{2 i n}^{*}(D) \geq S_{2}^{i *}(D)$ we denote:

$$
X_{2}^{i *}(D)=\frac{1}{k_{3} \alpha}\left(S_{2 i n}^{*}(D)-S_{2}^{i *}(D)\right), \quad i=1,2 .
$$

Lemma 1. The function $S_{1}^{*}(D)$ is increasing and the functions $X_{1}^{*}(D)$ and $S_{2 i n}^{*}(D)$ are decreasing on their domains of definition. The functions $S_{2}^{1 *}(D)$ and $S_{2}^{2 *}(D)$ are increasing and decreasing respectively. The functions $X_{2}^{1}(D)$ and $X_{2}^{1 *}(D)$ are decreasing on their domains of definition. The function $X_{2}^{2}(D)$ is increasing. The function $X_{2}^{2 *}(D)$ is not monotonous in general.

Proof 1. See proof in Appendix A.1. 
These properties of the functions are illustrated in Fig. 1. The functions $S_{1}^{*}(D), S_{2}^{i *}(D), X_{1}^{*}(D), X_{2}^{i}(D)$ and $X_{2}^{i *}(D)$ are depicted in this figure in the particular case of Monod and Haldane kinetics

$$
\mu_{1}\left(S_{1}\right)=\frac{m_{1} S_{1}}{K_{1}+S_{1}}, \quad \mu_{2}\left(S_{2}\right)=\frac{m_{2} S_{2}}{K_{2}+S_{2}+\frac{S_{2}^{2}}{K_{I}}},
$$

with the parameter values given in Table 1 . The bifurcations values $D_{1}, D_{2}$,

\begin{tabular}{|c|c|c|c|c|c|}
\hline$m_{1}=1.2$ & $K_{1}=2$ & $m_{2}=1.1$ & $K_{2}=10$ & $K_{I}=40$ & $k_{1}=25$ \\
\hline$k_{2}=250$ & $k_{3}=268$ & $\alpha=0.5$ & $S_{1 i n}=8$ & $S_{2 i n}=50$ & \\
\hline
\end{tabular}

Table 1: Nominal parameters values used for the generation of Figs. 1, 3 and 4.

$D_{3}$ and $D_{4}$ which appear in the figure are defined by:

$$
D_{1}=\frac{\mu_{1}\left(S_{1 i n}\right)}{\alpha}, \quad D_{2}=\frac{\mu_{2}^{M}}{\alpha}, \quad D_{3}=\frac{\mu_{2}\left(S_{2 i n}\right)}{\alpha}, \quad S_{2}^{2 *}\left(D_{4}\right)=S_{2 i n}^{*}\left(D_{4}\right) .
$$
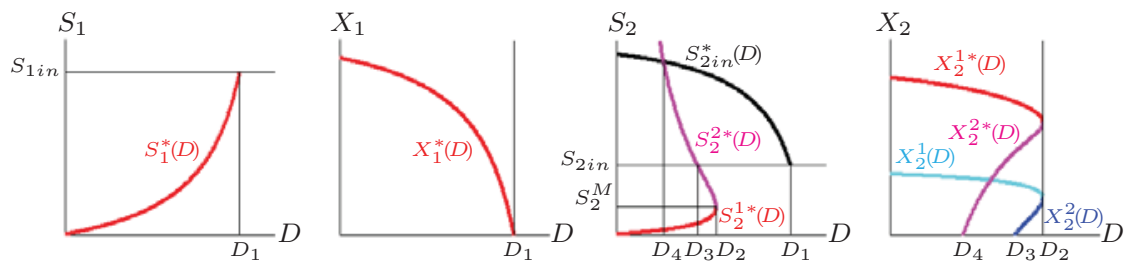

Figure 1: The graphs of the functions $S_{1}^{*}(D), X_{1}^{*}(D), S_{2}^{i *}(D), S_{2 i n}^{*}(D), X_{2}^{i}(D), X_{2}^{i *}(D)$, $i=1,2$. Here $D_{1}=1.92, D_{2}=1.1, D_{3} \simeq 0.89$ and $D_{4} \simeq 0.52$.

The equilibria of system (1-4) are solutions of the nonlinear algebraic system (6-9) obtained from (1-4) by setting the right-hand sides equal to zero:

$$
0=D\left(S_{1 i n}-S_{1}\right)-k_{1} \mu_{1}\left(S_{1}\right) X_{1}
$$




$$
\begin{aligned}
0 & =\left(\mu_{1}\left(S_{1}\right)-\alpha D\right) X_{1}, \\
0 & =D\left(S_{2 i n}-S_{2}\right)+k_{2} \mu_{1}\left(S_{1}\right) X_{1}-k_{3} \mu_{2}\left(S_{2}\right) X_{2}, \\
0 & =\left(\mu_{2}\left(S_{2}\right)-\alpha D\right) X_{2} .
\end{aligned}
$$

The calculation of the solutions of this set of equations is summarized in Fig. 2, (the details of the calculation is given in Appendix A.2).

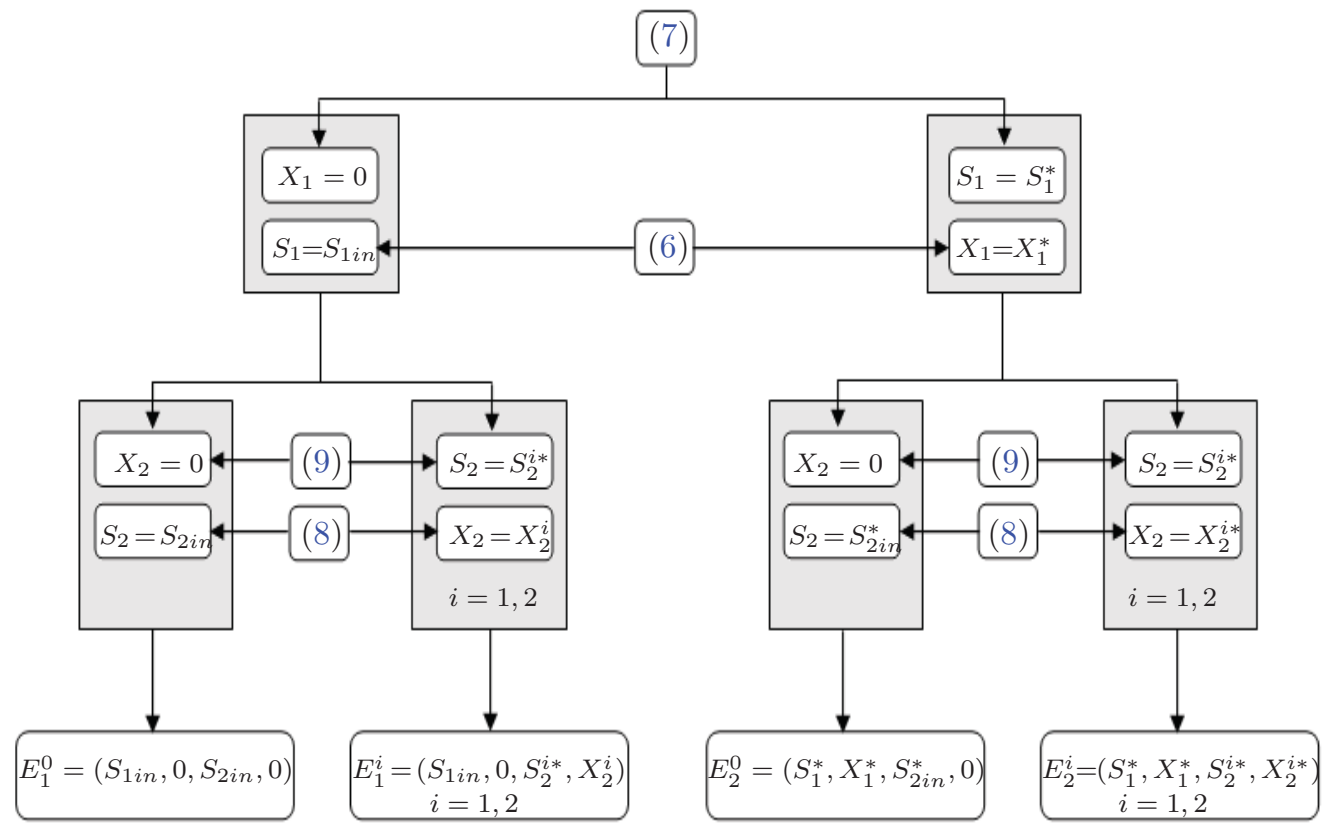

Figure 2: Diagram summarizing the equilibria of system (1-4). Hence, the system has at most six equilibrium points, see Proposition 1.

Proposition 1. System (1-4) has at most six equilibrium points:

$E_{1}^{0}=\left(S_{1 i n}, 0, S_{2 i n}, 0\right)$, which always exists and does not depend on $D$.

$E_{1}^{1}(D)=\left(S_{1 i n}, 0, S_{2}^{1 *}(D), X_{2}^{1}(D)\right)$, which exists if and only if $S_{2 i n} \geq S_{2}^{1 *}(D)$.

$E_{1}^{2}(D)=\left(S_{1 i n}, 0, S_{2}^{2 *}(D), X_{2}^{2}(D)\right)$, which exists if and only if $S_{2 i n} \geq S_{2}^{2 *}(D)$.

$E_{2}^{0}(D)=\left(S_{1}^{*}(D), X_{1}^{*}(D), S_{2 i n}^{*}(D), 0\right)$, which exists if and only if $S_{1 i n} \geq S_{1}^{*}(D)$. 
$E_{2}^{1}(D)=\left(S_{1}^{*}(D), X_{1}^{*}(D), S_{2}^{1 *}(D), X_{2}^{1 *}(D)\right)$, which exists if and only if $S_{1 \text { in }} \geq$ $S_{1}^{*}(D)$ and $S_{2 i n}^{*}(D) \geq S_{2}^{1 *}(D)$.

$E_{2}^{2}(D)=\left(S_{1}^{*}(D), X_{1}^{*}(D), S_{2}^{2 *}(D), X_{2}^{2 *}(D)\right)$, which exists if and only if $S_{1 \text { in }} \geq$ $S_{1}^{*}(D)$ and $S_{2 i n}^{*}(D) \geq S_{2}^{2 *}(D)$.

Proof 2. See proof in Appendix A.2.

The trivial equilibrium $E_{1}^{0}$ corresponds to the washout of $X_{1}$ and $X_{2}$. The trivial equilibria $E_{1}^{i}(D), i=1,2$ correspond to the washout of $X_{1}$ but not of $X_{2}$. The trivial equilibrium $E_{2}^{0}(D)$ corresponds to the washout of $X_{2}$ but not of $X_{1}$. The equilibria $E_{2}^{i}(D), i=1,2$ are positive. The first one, $E_{2}^{1}(D)$, is the nominal operating point, since it is locally stable. The second one, $E_{2}^{2}(D)$, is always unstable as shown in the following section. Since the nonconstant components of the equilibria are given by functions illustrated in Fig. 1, this figure represents also the equilibria of the system (see Figs. 3 and 4).

\subsection{Hyperbolic and Non-Hyperbolic equilibria}

The study of equilibria local stability follows easily from the study of the Jacobian matrix of system (1-4). Since there is no risk of confusion we drop the variable $D$ in the equilibria $E_{1}^{i}(D), E_{2}^{0}(D)$ and of $E_{2}^{i}(D)$ and their components. The existence and stability of the equilibria depend only on the relative positions of the values of $S_{1 \text { in }}$ and $S_{1}^{*}$ and of the values of $S_{2}^{1 *}, S_{2}^{2 *}, S_{2 i n}$, and $S_{2 i n}^{*}$. In the hyperbolic case, where the Jacobian matrix has eigenvalues whose real parts are different from 0 , the results on the existence and stability of the equilibria are summarized in Theorem 1 (cases 1.1 to 1.3 and 2.1 to 2.6 ). We list in each case the existing equilibria and their nature (where SE and UE stand for stable equilibrium and unstable 

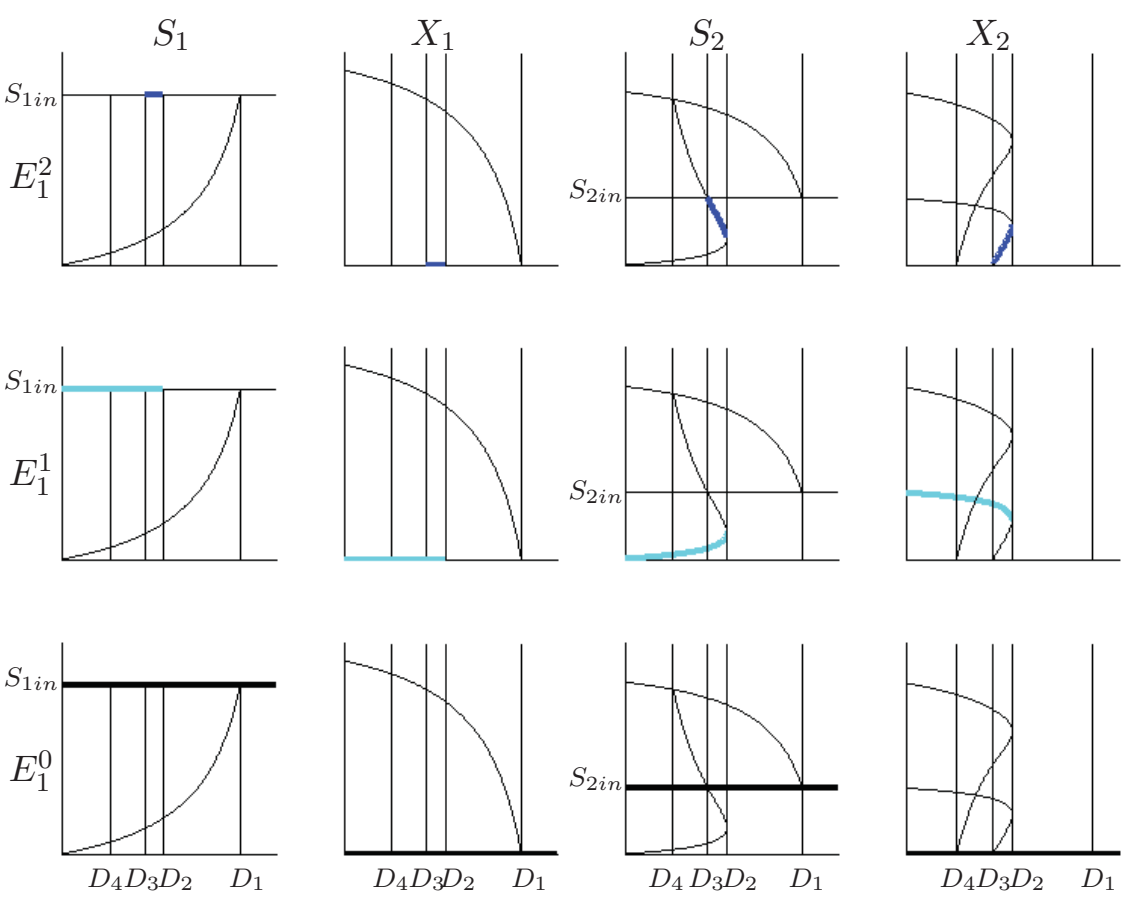

Figure 3: The components of the equilibrium points $E_{1}^{0}$ (in black), $E_{1}^{1}(D)$ (in cyan) and $E_{1}^{2}(D)$ (in blue) with respect to $D$. The vertical lines correspond to the values $D_{1}, D_{2}$, $D_{3}$ and $D_{4}$ used in Fig. 1.

equilibrium respectively).

When two of the four values $S_{2}^{1 *}(D), S_{2}^{2 *}(D), S_{2 i n}$, and $S_{2 i n}^{*}(D)$ are equal, then at least one of the eigenvalues of the Jacobian matrix has 0 as real part and we have a coalescence of equilibria. These cases are summarized in Theorem 1 (cases $\mathbf{1 . 4}$ to $\mathbf{1 . 6}$ and $\mathbf{2 . 7}$ to $\mathbf{2 . 1 5}$, where $\mathrm{NH}$ and $\mathrm{H}$ stand for Non Hyperbolic and Hyperbolic respectively).

\section{Theorem 1. Hyperbolic equilibria:}

If $S_{1 \text { in }}<S_{1}^{*}$ then we have 3 sub-cases: 

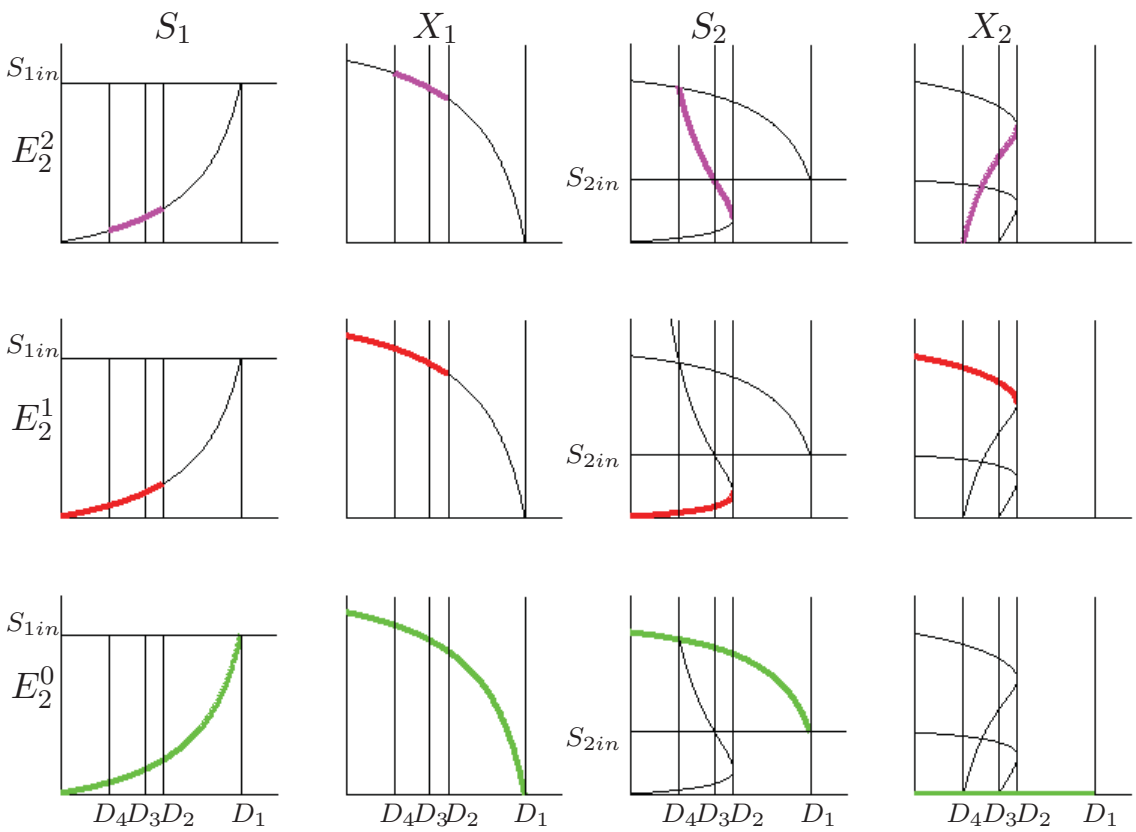

Figure 4: The components of the equilibrium points $E_{2}^{0}$ (in green), $E_{2}^{1}(D)$ (in red) and $E_{2}^{2}(D)$ (in magenta) with respect to $D$. The vertical lines correspond to the values $D_{1}$, $D_{2}, D_{3}$ and $D_{4}$ used in Fig. 1 .

\begin{tabular}{|l|c||c|c|c|}
\hline \multirow{2}{*}{ case } & \multicolumn{2}{|c||}{ Condition } & \multicolumn{3}{|c|}{ Equilibria and nature } \\
\cline { 3 - 5 } & & $E_{1}^{0}$ & $E_{1}^{1}$ & $E_{1}^{2}$ \\
\hline \hline $\mathbf{1 . 1}$ & $S_{2 \text { in }}<S_{2}^{1 *}<S_{2}^{2 *}$ & $S E$ & & \\
\hline $\mathbf{1 . 2}$ & $S_{2}^{1 *}<S_{2 i n}<S_{2}^{2 *}$ & $U E$ & $S E$ & \\
\hline $\mathbf{1 . 3}$ & $S_{2}^{1 *}<S_{2}^{2 *}<S_{2 i n}$ & $S E$ & $S E$ & $U E$ \\
\hline
\end{tabular}

If $S_{1 \text { in }}>S_{1}^{*}$ then we have 6 sub-cases: 


\begin{tabular}{|l|c||c|c|c|c|c|c|}
\hline \multirow{2}{*}{ case } & \multicolumn{1}{|c||}{ Condition } & \multicolumn{5}{|c|}{ Equilibria and nature } \\
\cline { 3 - 7 } & & $E_{1}^{0}$ & $E_{1}^{1}$ & $E_{1}^{2}$ & $E_{2}^{0}$ & $E_{2}^{1}$ & $E_{2}^{2}$ \\
\hline \hline $\mathbf{2 . 1}$ & $S_{2 i n}<S_{2 i n}^{*}<S_{2}^{1 *}<S_{2}^{2 *}$ & $U E$ & & & $S E$ & & \\
\hline $\mathbf{2 . 2}$ & $S_{2 i n}<S_{2}^{1 *}<S_{2 i n}^{*}<S_{2}^{2 *}$ & $U E$ & & & $U E$ & $S E$ & \\
\hline $\mathbf{2 . 3}$ & $S_{2 i n}<S_{2}^{1 *}<S_{2}^{2 *}<S_{2 i n}^{*}$ & $U E$ & & & $S E$ & $S E$ & $U E$ \\
\hline $\mathbf{2 . 4}$ & $S_{2}^{1 *}<S_{2 i n}<S_{2 i n}^{*}<S_{2}^{2 *}$ & $U E$ & $U E$ & & $U E$ & $S E$ & \\
\hline $\mathbf{2 . 5}$ & $S_{2}^{1 *}<S_{2 i n}<S_{2}^{2 *}<S_{2 i n}^{*}$ & $U E$ & $U E$ & & $S E$ & $S E$ & $U E$ \\
\hline $\mathbf{2 . 6}$ & $S_{2}^{1 *}<S_{2}^{2 *}<S_{2 i n}<S_{2 i n}^{*}$ & $U E$ & $U E$ & $U E$ & $S E$ & $S E$ & $U E$ \\
\hline
\end{tabular}

Non Hyperbolic equilibria:

If $S_{1 \text { in }}<S_{1}^{*}$, then we have 3 sub-cases:

\begin{tabular}{|l|c||c|l|}
\hline Case & Condition & NH Equilibria & H Equilibria \\
\hline \hline $\mathbf{1 . 4}$ & $S_{2 i n}=S_{2}^{1 *}<S_{2}^{2 *}$ & $E_{1}^{0}=E_{1}^{1}$ & \\
\hline $\mathbf{1 . 5}$ & $S_{2}^{1 *}<S_{2 i n}=S_{2}^{2 *}$ & $E_{1}^{0}=E_{1}^{2}$ & $E_{1}^{1}$ \\
\hline $\mathbf{1 . 6}$ & $S_{2}^{1 *}=S_{2}^{2 *}<S_{2 i n}$ & $E_{1}^{1}=E_{1}^{2}$ & $E_{1}^{0}$ \\
\hline
\end{tabular}

If $S_{1 \text { in }}>S_{1}^{*}$, then we have 9 sub-cases: 


\begin{tabular}{|c|c|c|c|}
\hline Case & Condition & NH Equilibria & H Equilibria \\
\hline 2.7 & $S_{2 i n}<S_{2 i n}^{*}=S_{2}^{1 *}<S_{2}^{2 *}$ & $E_{2}^{0}=E_{2}^{1}$ & $E_{1}^{0}$ \\
\hline 2.8 & $S_{2 i n}=S_{2}^{1 *}<S_{2 i n}^{*}<S_{2}^{2 *}$ & $E_{1}^{0}=E_{1}^{1}$ & $E_{2}^{0}, E_{2}^{1}$ \\
\hline 2.9 & $S_{2 i n}=S_{2}^{1 *}<S_{2}^{2 *}<S_{2 i n}^{*}$ & $E_{1}^{0}=E_{1}^{1}$ & $E_{2}^{1}, E_{2}^{2}, E_{2}^{0}$ \\
\hline 2.10 & $S_{2 i n}=S_{2}^{1 *}<S_{2 i n}^{*}=S_{2}^{2 *}$ & $\begin{array}{l}E_{1}^{0}=E_{1}^{1} \\
E_{2}^{0}=E_{2}^{2}\end{array}$ & $E_{2}^{1}$ \\
\hline 2.11 & $S_{2 i n}<S_{2}^{1 *}=S_{2}^{2 *}<S_{2 i n}^{*}$ & $E_{2}^{1}=E_{2}^{2}$ & $E_{1}^{0}, E_{2}^{0}$ \\
\hline 2.12 & $S_{2}^{1 *}<S_{2 i n}<S_{2 i n}^{*}=S_{2}^{2 *}$ & $E_{2}^{0}=E_{2}^{2}$ & $E_{1}^{0}, E_{1}^{1}, E_{2}^{1}$ \\
\hline 2.13 & $S_{2}^{1 *}<S_{2 i n}=S_{2}^{2 *}<S_{2 i n}^{*}$ & $E_{1}^{0}=E_{1}^{2}$ & $E_{1}^{1}, E_{2}^{1}, E_{2}^{0}, E_{2}^{2}$ \\
\hline 2.14 & $S_{2}^{1 *}=S_{2}^{2 *}<S_{2 i n}<S_{2 i n}^{*}$ & $\begin{array}{l}E_{1}^{1}=E_{1}^{2} \\
E_{2}^{1}=E_{2}^{2}\end{array}$ & $E_{1}^{0}, E_{2}^{0}$ \\
\hline 2.15 & $S_{2 i n}<S_{2}^{1 *}<S_{2 i n}^{*}=S_{2}^{2 *}$ & $E_{2}^{0}=E_{2}^{2}$ & $E_{1}^{0}, E_{2}^{1}$ \\
\hline
\end{tabular}

Proof 3. See proof in Appendix A.3.

Remark 1. [11] restricted their study to the case $S_{2 i n} \geq S_{2}^{M}\left({ }^{1}\right)$ arguing that otherwise, $\mu_{2}\left(S_{2}\right)$ should be monotonically increasing for $S_{2} \geq 0$. Actually, the condition $S_{2 i n}<S_{2}^{M}$ can lead to interesting phenomena, as the bistability of the system, which is not possible if $\mu_{2}\left(S_{2}\right)$ was monotonically increasing. The reason is that the bistability can arise if $S_{2 i n}^{*}>S_{2}^{M}$, even if $S_{2 i n}<S_{2}^{M}$ (see case 2.3 in Theorem 1).

\footnotetext{
${ }^{1}$ The authors mentioned that the Haldane model function $\mu_{2}\left(S_{2}\right)$ achieves a maximum at the point $S_{2}^{M}$, and they assumed that $S_{2}^{M} \leq S_{2 i n},\left(S_{2}^{M}\right.$ and $S_{2 i n}$ are noted $\tilde{s}_{2}$ and $s_{2}^{i}$ respectively in their paper). They noted that otherwise $\mu_{2}\left(S_{2}\right)$ would be monotonically increasing for $S_{2} \geq 0$ as Monod law $\mu_{1}\left(S_{1}\right)$ for $S_{1} \geq 0$ does.
} 


\subsection{Number of equilibria with respect to $D$}

From Theorem 1, we can easily deduce the number and nature of equilibria of the system with respect of the operating parameter $D$. For instance, Figs. 3 and 4 show the numbers of equilibria of (1-4) for the parameters values given in Table 1.

Of course, for other values of the parameters, the bifurcational values $D_{i}$ are not ranged as in the cases depicted in Fig. 1. For instance if all the parameters are as in Fig. 1 except for $K_{2}, K_{I}$ and $S_{1 \text { in }}$ which are set equal to 1,15 and 2 instead of 10, 40 and 8 respectively, then we have $D_{4}<D_{3}<D_{1}<D_{2}$ and not $D_{4}<D_{3}<D_{2}<D_{1}$, see Fig. 5 .
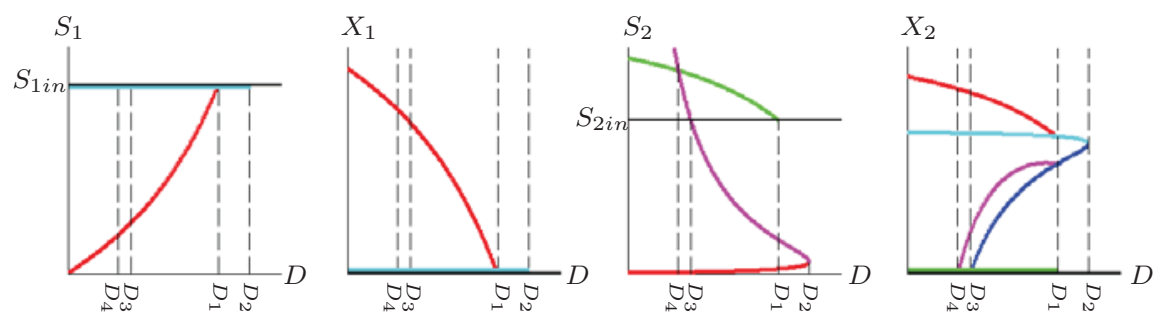

Figure 5: The components of the equilibrium points $E_{1}^{0}$ (in black), $E_{1}^{1}(D)$ and $E_{1}^{2}(D)$ (in cyan and blue respectively), $E_{2}^{0}(D)$ (in green) and $E_{2}^{1}(D)$ and $E_{2}^{2}(D)$ (in red and magenta respectively) with respect to $D$. Here $K_{2}=1, K_{I}=15$ and $S_{1 \text { in }}=2$ and hence $D_{1}=1.2$, $D_{2} \simeq 1.45, D_{3} \simeq 0.50$ and $D_{4} \simeq 0.40$.

We see on Fig. 5 that for $D=D_{1}$, there is an equilibria bifurcation:

$$
E_{2}^{1}\left(D_{1}\right)=E_{1}^{1}\left(D_{1}\right), \quad E_{2}^{2}\left(D_{1}\right)=E_{1}^{2}\left(D_{1}\right)
$$

This bifurcation was not considered in Theorem 1, since we assumed in this theorem that $S_{1 \text { in }} \neq S_{1}^{*}$. 


\subsection{Global behavior of model (1-4)}

Model (1-4) has a cascade structure which renders its analysis easy. We will take benefit of this structure to discuss the global behavior of the system. Following [12], page 430, we first remark that the system:

$$
\left\{\begin{array}{l}
\dot{S}_{1}=D\left(S_{1 \text { in }}-S_{1}\right)-k_{1} \mu_{1}\left(S_{1}\right) X_{1}, \\
\dot{X}_{1}=\left(\mu_{1}\left(S_{1}\right)-\alpha D\right) X_{1},
\end{array}\right.
$$

composed by (1) and (2) can be run autonomously. In other terms, $S_{1}$ and $X_{1}$ are not influenced by the other variables. Similarly the system composed by (3) and (4) can also be considered independently as a system taking its input from (10):

$$
\left\{\begin{array}{l}
\dot{S}_{2}=D\left(f(t)-S_{2}\right)-k_{3} \mu_{2}\left(S_{2}\right) X_{2}, \\
\dot{X}_{2}=\left(\mu_{2}\left(S_{2}\right)-\alpha D\right) X_{2},
\end{array}\right.
$$

with

$$
f(t)=S_{2 i n}+\frac{k_{2}}{D} \mu_{1}\left(S_{1}(t)\right) X_{1}(t),
$$

where $\left(S_{1}(t), X_{1}(t)\right)$ is a solution of $(10)$.

System (10) corresponds to a classical chemostat model with Monodkinetics type and specific mortality rate for $X_{1}$. The behavior of this system is well-known (cf. [4]). When $S_{1 \text { in }}>S_{1}^{*}(D)$, the nontrivial equilibrium is globally stable and the washout equilibrium $\left(X_{1}=0\right)$ is unstable. We restrict our analysis to this case. The case where the washout equilibrium is globally stable can be treated in the same way. Since the limit of $f(t)$ when $t \rightarrow+\infty$ exists and is equal to $S_{2 i n}^{*}$, system (11) converges to:

$$
\left\{\begin{array}{l}
\dot{S}_{2}=D\left(S_{2 i n}^{*}-S_{2}\right)-k_{3} \mu_{2}\left(S_{2}\right) X_{2}, \\
\dot{X}_{2}=\left(\mu_{2}\left(S_{2}\right)-\alpha D\right) X_{2} .
\end{array}\right.
$$


System (13) corresponds to a classical chemostat model with Haldane-type kinetics and specific mortality rate for $X_{2}$. The behavior of such a system is also well-known (cf. [4]). In the general case, it has three equilibria: the first one is positive and locally stable, the second one is positive and unstable and the third one is the locally stable trivial equilibrium (washout which corresponds to $X_{2}=0$ ). Now, the behavior of (1-4) follows from the following heuristic. Once the system (10) has converged to its equilibrium, the system (11) will also converge towards one of the two stable equilibria of (13) as it is illustrated in Fig. 6. This argument can be rigorously justified by using [5] results on asymptotically autonomous systems, see also [4] for the details of this kind of arguments.

Theorem 2. Assume that $S_{1 \text { in }}>S_{1}^{*}$ and $S_{2}^{1 *}<S_{2}^{2 *}<S_{2 i n}^{*}$, then all solutions of system (1-4) converge to either $E_{2}^{0}, E_{2}^{1}$ or $E_{2}^{2}$ and the set of initial conditions of solutions converging towards the saddle point $E_{2}^{2}$ is of measure 0. More precisely, the attractive stable manifold of $E_{2}^{2}$ is a 3-dimensional hypersurface which separates the phase space of (1-4) into the basins of attractions of the stable equilibria $E_{2}^{0}$ and $E_{2}^{1}$.

Proof 4. See proof in Appendix A.4.

We show in the next section how the previous generic analysis, can be used to revisit an interesting monitoring tool initially proposed by [9].

\section{Overloading tolerance}

\subsection{The overloading tolerance of Hess and Olivier}

In their paper on the analysis of the system (1-4), J. Hess and O. Bernard [9] noticed that for all solutions $\left(S_{1}(t), X_{1}(t)\right)$ of (10) we have $f(t) \leqslant \tilde{S}_{2 i n}$, 

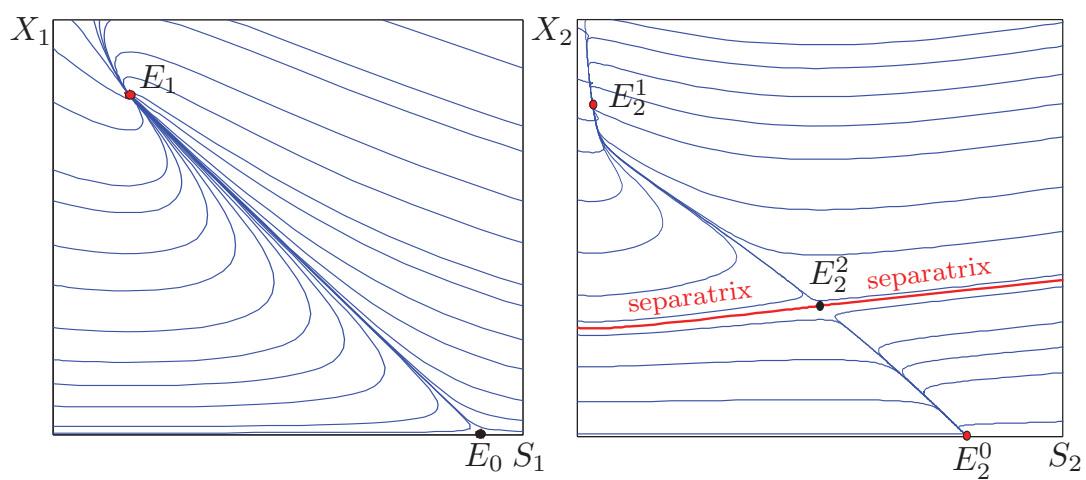

Figure 6: Left: Equilibria of system (10) where $E_{0}$ corresponds to the washout of $X_{1}$ and $E_{1}$ is the operating equilibrium $\left(S_{1}^{*}<S_{1 i n}\right)$. Right: Equilibria of system (13) where $E_{2}^{0}$ corresponds to the stable washout equilibrium of $X_{2}, E_{2}^{2}$ is the interior unstable equilibrium and $E_{2}^{1}$ is the interior stable equilibrium

where

$$
\tilde{S}_{2 i n}=S_{2 i n}+\frac{k_{2}}{k_{1}} S_{1 i n}
$$

and where $f(t)$ is defined by (12). Thus, the total input substrate concentration available for (11) is bounded by $\tilde{S}_{2 i n}$. In order to study the behavior of (11) they considered $\tilde{S}_{2 i n}$ as the "worst-case" upper bound of the total influent concentration in the reactor. Thus, they studied the equilibria of the system:

$$
\left\{\begin{array}{l}
\dot{S}_{2}=D\left(\tilde{S}_{2 i n}-S_{2}\right)-k_{3} \mu_{2}\left(S_{2}\right) X_{2}, \\
\dot{X}_{2}=\left(\mu_{2}\left(S_{2}\right)-\alpha D\right) X_{2} .
\end{array}\right.
$$

Notice that the equilibria of system (10), together with the equilibria of system (14) do not constitute the equilibria of the original system (1-4). In other words, the study they propose does not correspond to the study of the original system (1-4) as suggested in the literature. Notice also that there is no rigorous link between the solutions of (11) and those of (14). We will 
show here-below that considering (14) instead of (11) for monitoring a real process may be problematic under specific operating conditions. System (14) can have at most three equilibria:

- The trivial equilibrium $\xi_{2}^{0}=\left(\tilde{S}_{2 i n}, 0\right)$ corresponding to the washout of $X_{2}$;

- The positive equilibria $\xi_{2}^{i}(D)=\left(S_{2}^{i *}(D), \tilde{X}_{2}^{i}(D)\right), i=1,2$, where $\tilde{X}_{2}^{i}(D)=\frac{1}{k_{3} \alpha}\left(\tilde{S}_{2 i n}-S_{2}^{i *}(D)\right)$, which exists if and only if $\tilde{S}_{2 i n}>S_{2}^{i *}(D)$.

The stability of these equilibria depends on the relative positions of the values of $S_{2}^{1 *}(D), S_{2}^{2 *}(D)$, and $\tilde{S}_{2 i n}$, see Table 1 and Fig. 1 of [9]. A special case of interest is obtained when:

$$
S_{2}^{1 *}(D)<S_{2}^{2 *}(D)<\tilde{S}_{2 i n}
$$

Here $\xi_{2}^{0}$ and $\xi_{2}^{1}(D)$ are both stable and $\xi_{2}^{2}(D)$ is a saddle point whose separatrix separates the phase plane into the basins of attraction of $\xi_{2}^{0}$ and $\xi_{2}^{1}(D)$. To evaluate the size of the locally stable working point $\xi_{2}^{1}(D)$, [9] defined the overloading tolerance $(\mathrm{OT})$ as the distance between the equilibria $\xi_{2}^{1}(D)$ and $\xi_{2}^{2}(D):$

$$
M_{H B}(D)=\left\|\xi_{2}^{1}(D)-\xi_{2}^{2}(D)\right\| .
$$

Notice that they explicitly calculated $M_{H B}(D)$ only in the case of the Haldane kinetics. In fact, we can evaluate it in a more general case. Indeed, it may be shown that it depends only on the roots $S_{2}^{1 *}(D)$ and $S_{2}^{2 *}(D)$ of equation $\mu_{2}\left(S_{2}\right)=\alpha D$ and on the parameters $\alpha$ and $k_{3}$. More precisely, we have:

Proposition 2. The OT $M_{H B}(D)$ is given by:

$$
M_{H B}(D)=\left\|\xi_{2}^{1}(D)-\xi_{2}^{2}(D)\right\|=\sqrt{1+\frac{1}{k_{3}^{2} \alpha^{2}}}\left(S_{2}^{2 *}(D)-S_{2}^{1 *}(D)\right) .
$$


Proof 5. See proof in Appendix A.5.

The maximum value of the $\mathrm{OT}$ is denoted by $M_{c H B}(D)$. It is obtained when $\alpha D=\mu_{2}\left(\tilde{S}_{2 i n}\right)$. In this case we have:

$$
S_{2}^{1 *}(D)=\tilde{S}_{2}(D), \quad S_{2}^{2 *}(D)=\tilde{S}_{2 i n},
$$

and $\xi_{2}^{2}(D)$ coalesces with $\xi_{2}^{0}$. Hence:

$$
M_{c H B}(D)=M_{H B}\left(\frac{\mu_{2}\left(\tilde{S}_{2 i n}\right)}{\alpha}\right)=\sqrt{1+\frac{1}{k_{3}^{2} \alpha^{2}}}\left(\tilde{S}_{2 i n}-\tilde{S}_{2}(D)\right) .
$$

[9] defined the relative overloading tolerance (ROT) as the ratio $M_{H B}(D) / M_{c H B}(D)$.

Then, they propose the following "risk-index" as an on-line indicator of the destabilization of the process:

$$
R_{H B}= \begin{cases}1 & \text { for } \alpha D>\mu_{2}^{M} \\ 1-\frac{M_{H B}(D)}{M_{c H B}(D)} & \text { for } \mu_{2}\left(\tilde{S}_{2 i n}\right) \leq \alpha D \leq \mu_{2}^{M} \\ 0 & \text { for } \alpha D<\mu_{2}\left(\tilde{S}_{2 i n}\right) .\end{cases}
$$

Notice that the relative overloading tolerance is:

$$
\frac{M_{H B}(D)}{M_{c H B}(D)}=\frac{S_{2}^{2 *}(D)-S_{2}^{1 *}(D)}{\tilde{S}_{2 i n}-\tilde{S}_{2}(D)} .
$$

Based on the formal analysis we have performed in the section 2, we show in the next section that this risk criterion can be problematic if the system is underloaded.

\subsection{Comparison with the behavior of system (1-4)}

Operating conditions 1: Assume that:

$$
S_{2}^{1 *}(D)<S_{2}^{2 *}(D)<S_{2 i n}^{*}(D)<\tilde{S}_{2 i n} .
$$


This condition is compatible with (15). Thus, either the case $\mathbf{2 . 3}, \mathbf{2 . 5}$ or 2.6 holds and the system (1-4) has two stable equilibria $E_{2}^{0}(D)$ and $E_{2}^{1}(D)$, and one unstable equilibrium $E_{2}^{2}(D)$. Notice that $\tilde{X}_{2}^{i}(D)>X_{2}^{i *}(D)$. If one considers the equilibria $\xi_{2}^{0}, \xi_{2}^{1}(D)$, and $\xi_{2}^{2}(D)$ of system (14) instead of the actual equilibria $E_{2}^{0}(D), E_{2}^{1}(D)$, and $E_{2}^{2}(D)$ of system (1-4), then at equilibrium $\xi_{2}^{0}$ the substrate $S_{2}$ is overrated (since $\tilde{S}_{2 i n}>S_{2 i n}^{*}(D)$ ) and at equilibria $\xi_{2}^{1}(D)$ and $\xi_{2}^{2}(D)$ the biomass $X_{2}$ is overrated (since $\tilde{X}_{2}^{i}(D)>$ $\left.X_{2}^{i *}(D)\right)$, see Fig. 7 .

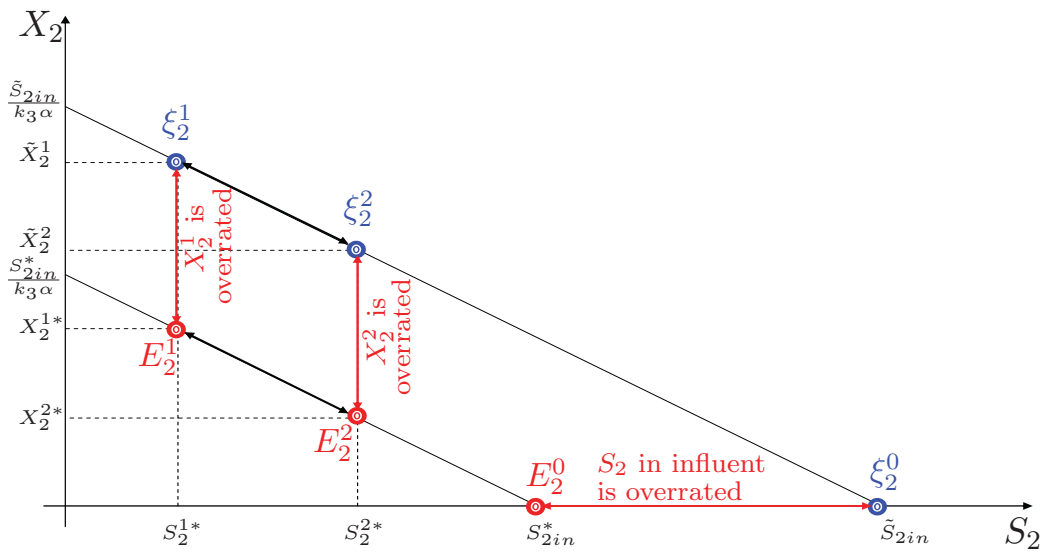

Figure 7: Equilibria $E_{2}^{0}(D), E_{2}^{1}(D)$, and $E_{2}^{2}(D)$ of $(1-4)$ and equilibria $\xi_{2}^{0}, \xi_{2}^{1}(D)$, and $\xi_{2}^{2}(D)$ of (14) under the condition (17).

We have $\tilde{X}_{2}^{i}(D)-X_{2}^{i *}(D)=\frac{\tilde{S}_{2 i n}-S_{2 i n}^{*}(D)}{k_{3} \alpha}=\frac{k_{2} S_{1}^{*}}{k_{1} k_{3} \alpha}$ for $i=1,2$. Hence, the distance between the equilibria $E_{2}^{1}(D)$ and $E_{2}^{2}(D)$ is the same as the distance between the equilibria $\xi_{2}^{1}(D)$ and $\xi_{2}^{2}(D)$. Thus, the OT is also given by $M(D)=\left\|E_{2}^{1}(D)-E_{2}^{2}(D)\right\|$. The first conclusion we draw from this reasoning is that if (17) holds, [9] would have obtained the same criterion if they had considered the actual equilibria $E_{2}^{1}(D)$ and $E_{2}^{2}(D)$ of system (1-4) rather 
than equilibria $\xi_{2}^{1}(D)$ and $\xi_{2}^{2}(D)$ of system (14).

Operating conditions 2: Assume now that:

$$
S_{2}^{1 *}(D)<S_{2 i n}^{*}(D)<S_{2}^{2 *}(D)<\tilde{S}_{2 i n}
$$

Either the case $\mathbf{2 . 2}$ or $\mathbf{2 . 4}$ holds and the system (1-4) has one stable equilibrium $E_{2}^{1}(D)$ and one unstable equilibrium $E_{2}^{0}(D)$. However, the system (14) has three equilibria $\xi_{2}^{0}, \xi_{2}^{1}(D)$, and $\xi_{2}^{2}(D)$, see Fig. 8 on the left. In this case the OT criterion, which has been developed for a bistable system, is not appropriate since the actual system has only one stable equilibrium.

The following case is even more problematic if the process is operated on the basis of the OT criterion.

Operating conditions 3: Let:

$$
S_{2 i n}^{*}(D)<S_{2}^{1 *}(D)<S_{2}^{2 *}(D)<\tilde{S}_{2 i n}
$$

In practice, it means that there is an "underload" of $S_{2}$ into the system. In other words, there is not enough substrate $S_{2}$ for $X_{2}$ to grow. Thus, the case 2.1 holds and the system (1-4) has only one stable equilibrium $E_{2}^{0}(D)$ which corresponds to the washout of $X_{2}$. However, the system (14) has three equilibria $\xi_{2}^{0}, \xi_{2}^{1}(D)$, and $\xi_{2}^{2}(D)$, see Fig. 8 on the right. In this case, the use of the OT criterion to assess the management of the process would not give the right information to the user. Indeed, the OT criterion asserts that the system is functioning near the stable positive equilibrium while the actual behavior of the real system leads to the washout of $X_{2}$.

\subsection{Risk index and numerical simulations}

To solve the problem we have just pointed out, we propose to modify the OT and the risk-index originally proposed by [9] as follows. Assume that 

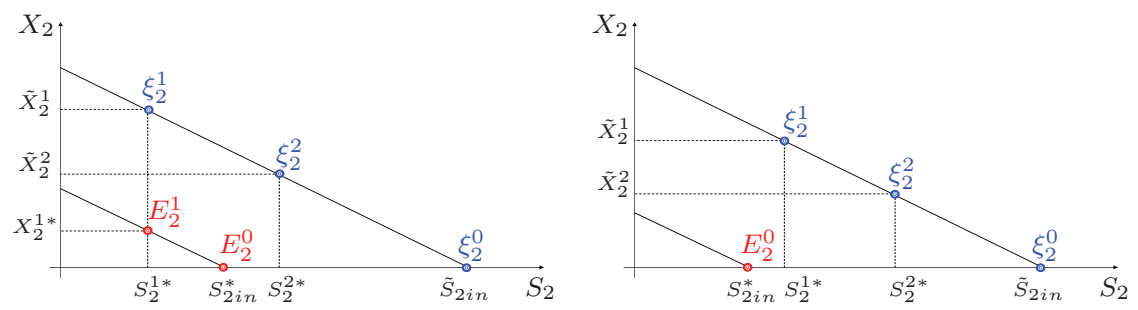

Figure 8: On the left, equilibria $E_{2}^{0}(D)$ and $E_{2}^{1}(D)$ of (1-4) under the condition (18). On the right, equilibrium $E_{2}^{0}(D)$ of (1-4) under the condition (19).

$S_{2 \text { in }}^{*}(D)>S_{2}^{M}$. Let $\alpha D \in\left[\mu_{2}\left(S_{2 i n}^{*}(D)\right), \mu_{2}^{M}\right]$. Then (1-4) has three equilibria $E_{2}^{0}(D), E_{2}^{1}(D)$, and $E_{2}^{2}(D)$. We define the OT (noted hereafter $M(D)$ ) as the distance between $E_{2}^{1}(D)$ and $E_{2}^{2}(D)$ :

$$
M(D)=\left\|E_{2}^{1}(D)-E_{2}^{2}(D)\right\|
$$

More precisely, we have:

Proposition 3. The OT $M(D)$ is given by:

$$
M(D)=\left\|E_{2}^{1}(D)-E_{2}^{2}(D)\right\|=\sqrt{1+\frac{1}{k_{3}^{2} \alpha^{2}}}\left(S_{2}^{2 *}(D)-S_{2}^{1 *}(D)\right) .
$$

Proof 6. See proof in Appendix A.6.

The maximum value of $M(D)$ is denoted by $M_{c}(D)$. It is obtained when $\alpha D=\mu_{2}\left(S_{2 i n}^{*}(D)\right)$. In this case we have:

$$
S_{2}^{1 *}(D)=S_{2}^{*}(D), \quad S_{2}^{2 *}(D)=S_{2 i n}^{*}(D),
$$

and $E_{2}^{2}(D)$ coalesces with $E_{2}^{0}(D)$. Hence:

$$
M_{c}(D)=M\left(\frac{\mu_{2}\left(S_{2 i n}^{*}(D)\right)}{\alpha}\right)=\sqrt{1+\frac{1}{k_{3}^{2} \alpha^{2}}}\left(S_{2 i n}^{*}(D)-S_{2}^{*}(D)\right) .
$$


In Fig. 9, we show the proposed and the modified margins.

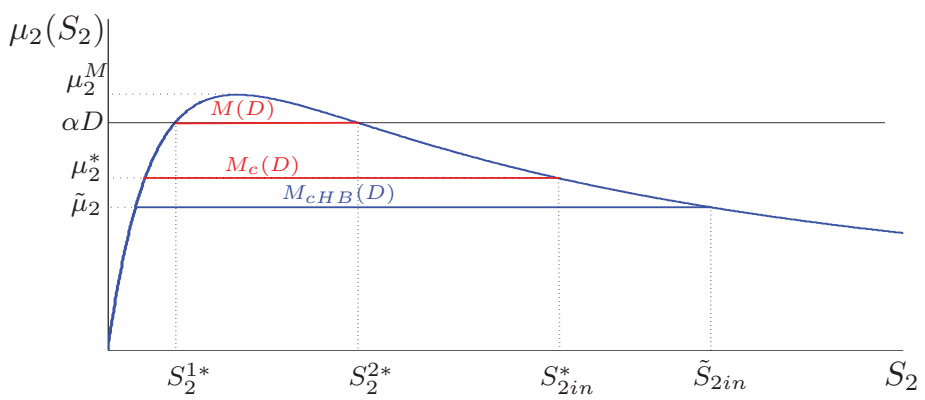

Figure 9: Proposed and modified OT with $\mu_{2}^{*}=\mu_{2}\left(S_{2 i n}^{*}\right)$ and $\tilde{\mu}_{2}=\mu_{2}\left(\tilde{S}_{2 i n}\right)$.

We define the risk-index as follows:

$$
R= \begin{cases}1 & \text { for } S_{2 i n}^{*}(D)<S_{2}^{1 *}(D) \text { or } \alpha D>\mu_{2}^{M}, \\ 1-\frac{M(D)}{M_{c}(D)} & \text { for } S_{2}^{2 *}(D)<S_{2 i n}^{*}(D), \\ 0 & \text { for } S_{2}^{1 *}(D)<S_{2 i n}^{*}(D)<S_{2}^{2 *}(D) .\end{cases}
$$

Notice that the relative overloading tolerance is now given by:

$$
\frac{M(D)}{M_{c}(D)}=\frac{S_{2}^{2 *}(D)-S_{2}^{1 *}(D)}{S_{2 i n}^{*}(D)-S_{2}^{*}(D)}
$$

Let us illustrate our approach in simulation. In particular, we compare the risk-index (16) of [9] and our risk-index (20) for the model (1-4) with Monod and Haldane kinetics (5), and nominal parameter values close to the parameters of [12], (cf. Table 2). Let us notice that we changed some values to have the bistability behaviour of the system, because with the default values of [12], the system is always working only around functional equilibrium. The values of the operating parameters are

$$
D=0.8 d^{-1}, \quad S_{1 i n}=10 \mathrm{~g} / L, \quad S_{2 i n}=5 \mathrm{mmol} / \mathrm{L} .
$$




\begin{tabular}{|c|c|c|c|c|}
\hline Parameter & Unit & Value of [12] & Standard Deviation & Nominal value \\
\hline \hline$m_{1}$ & $d^{-1}$ & 1.2 & & 1.2 or 0.5 \\
$K_{1}$ & $g / L$ & 7.1 & 5 & 2.1 \\
$m_{2}$ & $d^{-1}$ & 0.74 & 0.9 & 0.95 \\
$K_{2}$ & $m m o l / L$ & 9.28 & 13.7 & 24 \\
$K_{i}$ & $m m o l / L$ & 256 & 320 & 55 \\
$\alpha$ & & 0.5 & 0.4 & 0.5 \\
$k_{1}$ & & 42.14 & 18.94 & 25 \\
$k_{2}$ & $m m o l / g$ & 116.5 & 113.6 & 250 \\
$k_{3}$ & $m m o l / g$ & 268 & 52.31 & 268 \\
\hline
\end{tabular}

Table 2: Nominal parameters values.

We consider a first period of time $0 \leq t \leq 50$ such as $m_{1}=1.2$ and, a second period $50 \leq t \leq t_{\text {final }}$ such as $m_{1}$ changes its value to 0.5 . The objective is to illustrate different situations where $R$ can change while $R_{H B}$ remains constant.

When $m_{1}=1.2$ we have, (see Fig. 10 on the left and Fig. 11 on the left),

$$
S_{1}^{*}=1.05, \quad S_{2}^{1 *}(D)=27.33, \quad S_{2}^{2 *}(D)=48.29, \quad S_{2 i n}^{*}(D)=94.5 .
$$

Thus, case $\mathbf{2 . 3}$ holds and the system has four equilibria:

$$
\begin{gathered}
E_{1}^{0}=(10,0,5,0), \quad E_{2}^{0}(D)=(1.05,0.72,94.5,0), \\
E_{2}^{1}(D)=(1.05,0.72,27.33,0.5), \quad E_{2}^{2}(D)=(1.05,0.72,48.29,0.34) .
\end{gathered}
$$

This is the bistability case. The value of the $R$ index is 0.74 which indicates that the destabilization risk is present. Notice that $\tilde{S}_{2 i n}=105$. The value of 

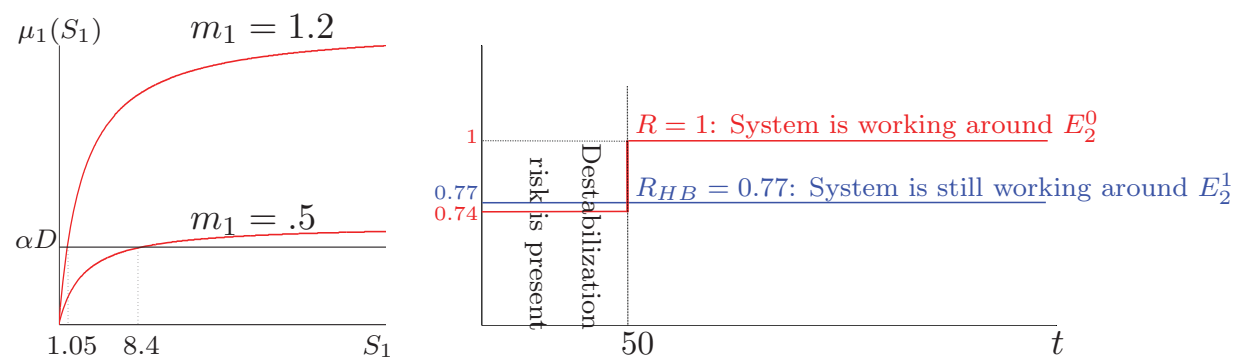

Figure 10: On the left, the function $\mu_{1}\left(S_{1}\right)$ for $m_{1}=0.5$ and $m_{1}=1.2$. On the right, the risks $R_{H B}$ and $R$ according to $t$. At time $t=50$, the parameter $m_{1}$ changes from 1.2 to 0.5 .
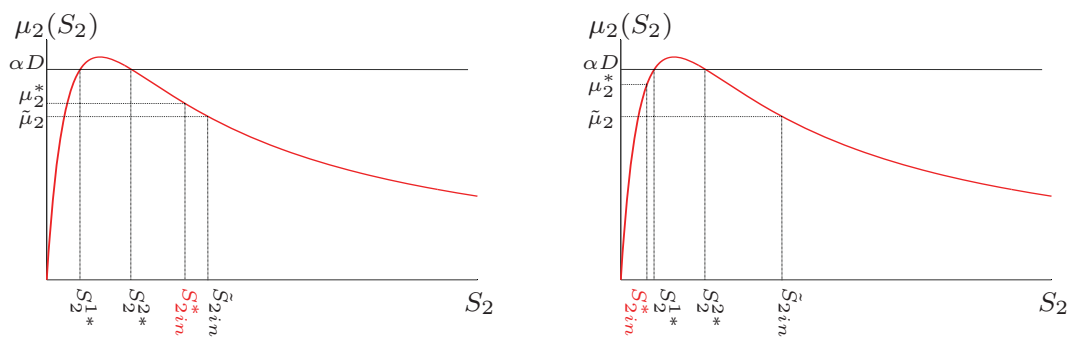

Figure 11: On the left, the function $\mu_{2}\left(S_{2}\right)$ for $m_{1}=1.2$. On the right, the function $\mu_{2}\left(S_{2}\right)$ for $m_{1}=0.5$. With $\mu_{2}^{*}=\mu_{2}\left(S_{2 i n}^{*}(D)\right)$ and $\tilde{\mu}_{2}=\mu_{2}\left(\tilde{S}_{2 i n}\right)$

the $R_{H B}$ index is 0.77 which leads also to the same conclusion. This is the situation depicted in Fig. 7 . When $m_{1}=0.5$ we have (see Fig. 10 on the left and Fig. 11 on the right),

$$
S_{1}^{*}=8.4, \quad S_{2}^{1 *}(D)=27.33, \quad S_{2}^{2 *}(D)=48.29, \quad S_{2 i n}^{*}(D)=21 .
$$

Thus the case $\mathbf{2 . 1}$ holds and the system has only two equilibria:

$$
E_{1}^{0}=(10,0,5,0), \quad E_{2}^{0}(D)=(8.4,0.13,21,0) .
$$

This is the washout case. The value of the $R$ index is then 1 which indicates that the destabilization risk is very important. Since the value of $\tilde{S}_{2 i n}=105$ 
has not changed, the value of the $R_{H B}$ index is 0.77 which clearly leads to a bad conclusion. This is the situation depicted in Fig. 8 on the right.
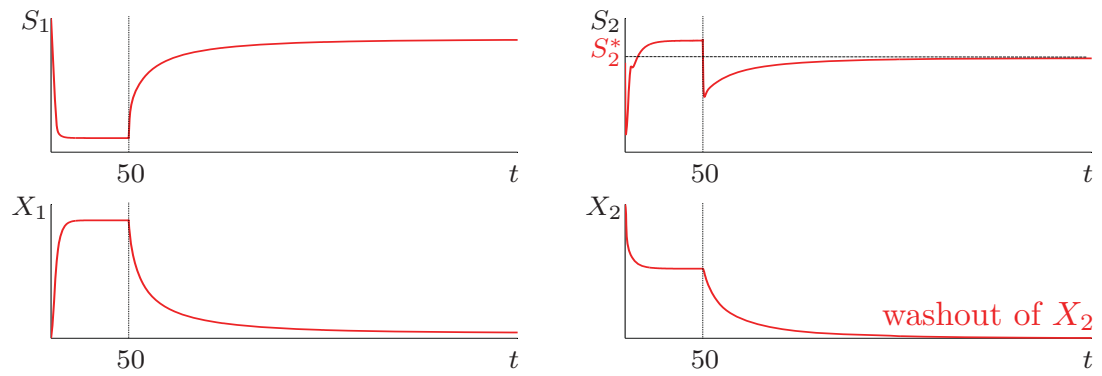

Figure 12: At time $t=50$ the parameter $m_{1}$ is changed from 1.2 to 0.5 . The solutions $S_{1}(t), X_{1}(t), S_{2}(t)$ and $X_{2}(t)$ according to $t$, showing that $X_{2}(t) \rightarrow 0$.

At time $t=50$ the parameter $m_{1}$ is changed from its nominal value 1.2 to a nominal value 0.5 , see Fig. 12. The simulation is carried out using initial conditions:

$$
S_{1}(0)=10, \quad X_{1}(0)=0.1, \quad S_{2}(0)=20, \quad X_{2}(0)=1 .
$$

The index $R_{H B}$ does not change at time $t=50$ and remains equal to its value 0.77 since this index does not depend on the parameters of the $\mu_{1}$ kinetics. However, at time $t=50$ the index $R$ jumps from its value 0.74 to the value 1 (see Fig. 10 on the right), which indicates to the operator that something bad is going to occur (see Fig. 12).

At this step, it may be argued that the actual proposed OT modification requires an accurate monitoring and a quite important a-priori knowledge about the system. In particular, regarding the definition of $S_{2 i n}^{*}$, accurate information about the acidogenic step (the $1^{\text {st }}$ reaction) is required. However, we argue that it is only at this price that the process can be operated properly. 


\section{Conclusion}

The paper has presented a complete mathematical analysis of the two step anaerobic model initially proposed by [12]. Under certain operating conditions, we have highlighted that the overloading tolerance parameter based on this model and initially proposed by [9] could lead to bad decisions and thus could not be used. Based on our analysis, we revisited the OT in order to capture the right information when the process is operating in a bistable mode.

Perspectives of this work include (i) the search for observers to estimate the required information for the new proposed monitoring strategy to be applied from simple available on-line information and (ii) the extension of these qualitative results to the model proposed in [1] to take into account the presence of soluble microbial products which seem to play an important role in a number of advanced treatment processes such as membranes biorectors.

\section{Appendix A. Proofs}

In this section we give the proofs of the mathematical results.

\section{Appendix A.1. Proof of Lemma 1}

Using the implicit function theorem one obtains that:

$$
S_{1}^{* \prime}(D)=\frac{\alpha}{\mu_{1}^{\prime}\left(S_{1}^{*}\right)} .
$$

Using Hypothesis 1 one obtains $S_{1}^{* \prime}(D)>0$. Thus $S_{1}^{*}$ is increasing. One has:

$$
S_{2 i n}^{*}{ }^{\prime}(D)=-\frac{k_{2}}{k_{1}} S_{1}^{* \prime}(D), \quad X_{1}^{* \prime}(D)=-\frac{1}{k_{1} \alpha} S_{1}^{* \prime}(D) .
$$


Thus $X_{1}^{*}(D)$ and $S_{2 i n}^{*}(D)$ are decreasing. Similarly, using the implicit function theorem one obtains that:

$$
S_{2}^{i *^{\prime}}(D)=\frac{\alpha}{\mu_{2}^{\prime}\left(S_{2}^{i *}(D)\right)} .
$$

Since $0<S_{2}^{1 *}(D) \leq S_{2}^{M} \leq S_{2}^{2 *}(D)$ and using Hypothesis 2 one obtains $S_{2}^{1 *^{\prime}}(D)>0$ and $S_{2}^{2 *^{\prime}}(D)<0$. Thus $S_{2}^{1 *}(D)$ is increasing $S_{2}^{2 *}(D)$ is decreasing. One has:

$$
\begin{gathered}
X_{2}^{i^{\prime}}(D)=-\frac{1}{k_{3} \alpha} S_{2}^{i *^{\prime}}(D), \quad i=1,2 ; \\
X_{2}^{1 *^{\prime}}(D)=-\frac{1}{k_{3} \alpha}\left(\frac{k_{2}}{k_{1}} S_{1}^{*^{\prime}}(D)+S_{2}^{1 *^{\prime}}(D)\right) .
\end{gathered}
$$

Thus $X_{2}^{1}(D)$ and $X_{2}^{1 *}(D)$ are decreasing and $X_{2}^{2}(D)$ is increasing. Notice that:

$$
X_{2}^{2 *^{\prime}}(D)=-\frac{1}{k_{3} \alpha}\left(\frac{k_{2}}{k_{1}} S_{1}^{*^{\prime}}(D)+S_{2}^{2 *^{\prime}}(D)\right) .
$$

is the sum of a positive and a negative function. Thus $X_{2}^{2 *}(D)$ is not monotonous. This ends the proof of the lemma.

\section{Appendix A.2. Proof of Proposition 1}

The equilibrium points are solutions of the nonlinear algebraic system (6-9). We are looking for nonnegative solutions $\left(S_{1}, X_{1}, S_{2}, X_{2}\right)$ of the above system. Equation (7) possesses the trivial solution:

$$
X_{1}=0,
$$

and the nontrivial solution:

$$
S_{1}=S_{1}^{*}(D)
$$


Then solving (6) with (A.1) leads to:

$$
S_{1}=S_{1 i n}
$$

and solving (6) with (A.2) leads to:

$$
X_{1}=\frac{1}{k_{1} \alpha}\left(S_{1 i n}-S_{1}^{*}(D)\right)=X_{1}^{*}(D),
$$

which is nonnegative if and only if $S_{1 i n} \geq S_{1}^{*}(D)$. Equation (9) possesses the trival solution:

$$
X_{2}=0 \text {, }
$$

and the nontrivial solutions:

$$
S_{2}=S_{2}^{i *}(D), \quad i=1,2
$$

Then solving (8) with (A.1), (A.3) and (A.5) leads to:

$$
S_{2}=S_{2 i n}
$$

The values (A.1), (A.3), (A.5) and (A.7) are the components of $E_{1}^{0}$. Similarly, solving (8) with (A.1), (A.3) and (A.6) leads to:

$$
X_{2}=\frac{1}{k_{3} \alpha}\left(S_{2 i n}-S_{2}^{i *}(D)\right)=X_{2}^{i}(D), \quad i=1,2 ;
$$

which is nonnegative if and only if $S_{2}^{i *}(D) \leq S_{2 i n}$. The values (A.1), (A.3), (A.6) and (A.8) are the components of $E_{1}^{i}(D)$. On the other hand, solving (8) with (A.2), (A.4) and (A.5) leads to:

$$
S_{2}=S_{2 i n}+k_{2} \alpha X_{1}^{*}(D)=S_{2 i n}^{*}(D) .
$$

The values (A.2), (A.4) and (A.5) and (A.9) are the components of $E_{2}^{0}(D)$. Similarly, solving (8) with (A.2), (A.4) and (A.6) leads to:

$$
X_{2}=\frac{1}{k_{3} \alpha}\left(S_{2 i n}^{*}(D)-S_{2}^{i *}(D)\right)=X_{2}^{i *}(D), \quad i=1,2,
$$


which are nonnegative if and only if $S_{2}^{i *}(D) \leq S_{2 i n}^{*}(D)$. The values (A.2), (A.4), (A.6) and (A.10) are the components of $E_{2}^{i}(D)$. This ends the proof of the proposition.

\section{Appendix A.3. Proof of Theorem 1}

\section{Hyperbolic equilibria:}

We compute the Jacobian matrix of system (1-4). This matrix as a blockdiagonal structure:

$$
J=\left[\begin{array}{ll}
A & 0 \\
C & B
\end{array}\right],
$$

where:

$$
\begin{aligned}
& A=\left[\begin{array}{cc}
-D-k_{1} \mu_{1}^{\prime}\left(S_{1}\right) X_{1} & -k_{1} \mu_{1}\left(S_{1}\right) \\
\mu_{1}^{\prime}\left(S_{1}\right) X_{1} & \mu_{1}\left(S_{1}\right)-\alpha D
\end{array}\right], \\
& B=\left[\begin{array}{cc}
-D-k_{3} \mu_{2}^{\prime}\left(S_{2}\right) X_{2} & -k_{3} \mu_{2}\left(S_{2}\right) \\
\mu_{2}^{\prime}\left(S_{2}\right) X_{2} & \mu_{2}\left(S_{2}\right)-\alpha D
\end{array}\right] .
\end{aligned}
$$

Hence, the eigenvalues of $J$ are the eigenvalues of $A$ and the eigenvalues of B. Equilibria stability is summarized in Table A.3.

\section{Non Hyperbolic equilibria:}

Let us give the details of the proof in the case 2.12. The other cases can be studied similarly (see Fig. A.13).

Assume that $S_{2}^{1 *}(D)<S_{2 i n}<S_{2}^{2 *}(D)=S_{2 i n}^{*}(D)$ as shown in Fig. A.13, case 2.12 , then $X_{2}^{2 *}(D)=0, X_{2}^{1 *}(D)>0$ and $X_{2}^{1}(D)>0$. Therefore (see Proposition 1), the system has four equilibria $E_{1}^{0}, E_{1}^{1}(D), E_{2}^{1}(D)$ and $E_{2}^{2}(D)=E_{2}^{0}(D)$. Using the linearization as in proof of Hyperbolic Equilibria, we obtain that $E_{1}^{0}, E_{1}^{1}(D), E_{2}^{1}(D)$ are hyperbolic. 


\begin{tabular}{|c|c|c|c|c|}
\hline Equilibria & Evalu & uated matrices $A$ and & & Conditions of stability \\
\hline$E_{1}^{0}$ & $\begin{array}{l}A= \\
B=\end{array}$ & {$\left[\begin{array}{cc}-D & -k_{1} \mu_{1}\left(S_{1 i n}\right) \\
0 & \mu_{1}\left(S_{1 i n}\right)-\alpha D \\
-D & -k_{3} \mu_{2}\left(S_{2 i n}\right) \\
0 & \mu_{2}\left(S_{2 i n}\right)-\alpha D\end{array}\right]$} & & $\begin{array}{l}\operatorname{tr}(A)<0 \text { if } S_{1 i n}<S_{1}^{*}, \operatorname{det}(A)>0 \\
\operatorname{tr}(B)<0 \text { if } S_{2 i n}<S_{2}^{1 *} \text { or } S_{2 i n}>S_{2}^{2 *}, \operatorname{det}(B)>0 \\
\Rightarrow\left\{\begin{array}{cc}E_{1}^{0} \quad \text { is stable if } S_{1 i n}<S_{1}^{*} \text { and } \\
\quad\left(S_{2 i n}<S_{2}^{1 *} \text { or } S_{2 i n}>S_{2}^{2 *}\right), \\
E_{1}^{0} \quad \text { is unstable if } S_{1 i n}>S_{1}^{*} \text { or } \\
& \left(S_{1 i n}<S_{1}^{*} \text { and } S_{2}^{1 *}<S_{2 i n}<S_{2}^{2 *}\right) .\end{array}\right.\end{array}$ \\
\hline $\begin{array}{c}E_{1}^{i}, \\
i=1,2\end{array}$ & $\begin{array}{l}A= \\
B=\end{array}$ & 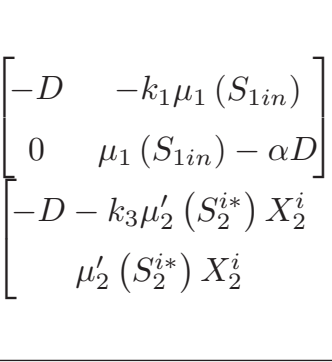 & $\left.\begin{array}{c}-k_{3} \alpha D \\
0\end{array}\right]$ & $\begin{array}{l}\operatorname{tr}(A)<0 \text { and } \operatorname{det}(A)>0 \text { if } S_{1 \text { in }}<S_{1}^{*} \\
\operatorname{tr}(B)<0 \text { and } \operatorname{det}(B)>0 \text { at } E_{1}^{1} \\
\operatorname{det}(B)<0 \text { at } E_{1}^{2} \\
\Rightarrow\left\{\begin{array}{cl}E_{1}^{1} \text { is stable, } \\
E_{1}^{2} \text { is unstable, } \\
E_{1}^{i} \text { are both unstable if } S_{1 \text { in }}>S_{1}^{*} .\end{array}\right.\end{array}$ \\
\hline$E_{2}^{0}$ & $\begin{array}{l}A= \\
B=\end{array}$ & {$\left[\begin{array}{cc}-D-k_{1} \mu_{1}^{\prime}\left(S_{1}^{*}\right) X_{1}^{*} \\
& \mu_{1}^{\prime}\left(S_{1}^{*}\right) X_{1}^{*} \\
-D & -k_{3} \mu_{2}\left(S_{2 i n}^{*}\right) \\
0 & \mu_{2}\left(S_{2 i n}^{*}\right)-\alpha D\end{array}\right]$} & {$\left[\begin{array}{c}-k_{1} \alpha D \\
0\end{array}\right]$} & $\begin{array}{l}\operatorname{tr}(A)<0 \text { and } \operatorname{det}(A)>0 \\
\operatorname{tr}(B)<0 \text { and } \operatorname{det}(B)>0 \text { if } S_{2 i n}^{*}<S_{2}^{1 *} \text { or } S_{2 i n}^{*}>S_{2}^{2 *} \\
\Rightarrow \begin{cases}E_{2}^{0} \quad \text { is stable if } S_{1 i n}>S_{1}^{*} \text { and } \\
& \left(S_{2 \text { in }}^{*}<S_{2}^{1 *} \text { or } S_{2 i n}^{*}>S_{2}^{2 *}\right) . \\
E_{2}^{0} \quad \text { is unstable if } S_{1 i n}>S_{1}^{*} \text { and } \\
& S_{2}^{1 *}<S_{2 i n}^{*}<S_{2}^{2 *} .\end{cases} \end{array}$ \\
\hline $\begin{array}{c}E_{2}^{i}, \\
i=1,2\end{array}$ & $\begin{array}{l}A= \\
B=\end{array}$ & 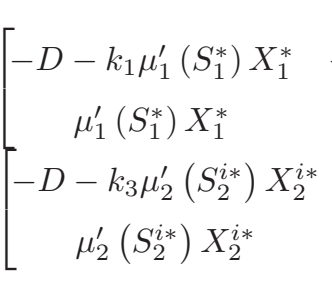 & $\left.\begin{array}{c}-k_{1} \alpha D \\
0 \\
-k_{3} \alpha D \\
0\end{array}\right]$ & $\begin{array}{l}\operatorname{tr}(A)<0 \text { and } \operatorname{det}(A)>0 \\
\operatorname{tr}(B)<0 \text { and } \operatorname{det}(B)>0 \text { at } E_{2}^{1} \\
\operatorname{det}(B)<0 \text { at } E_{2}^{2} \\
\Rightarrow \begin{cases}E_{2}^{1} \text { is stable, } \\
E_{2}^{2} \text { is unstable. }\end{cases} \end{array}$ \\
\hline
\end{tabular}

Table A.3: Stability of hyperbolic equilibria of the system (1-4) 

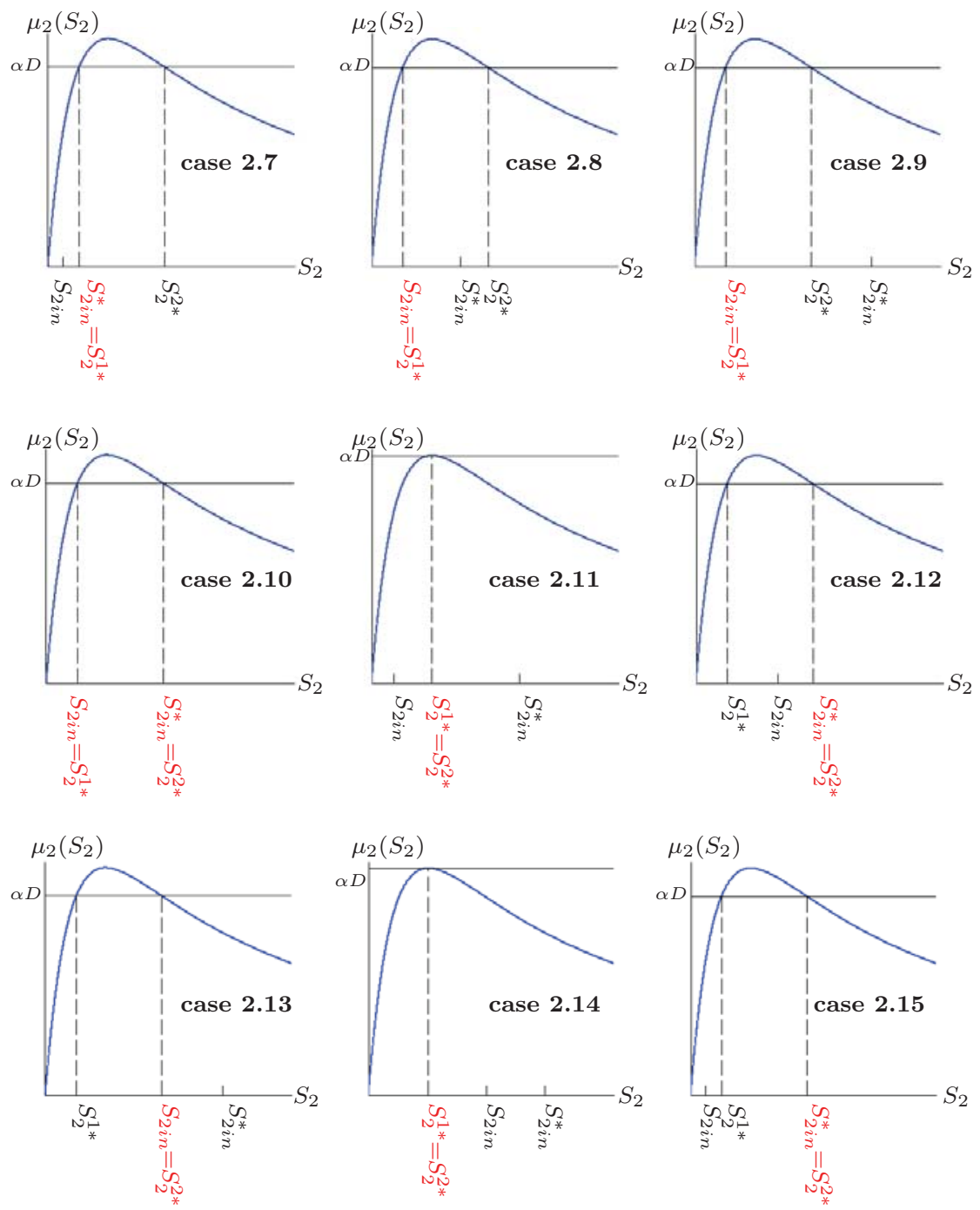

Figure A.13: Different cases when two of the four values $S_{2}^{1 *}(D), S_{2}^{2 *}(D), S_{2 \text { in }}$ and $S_{2 \text { in }}^{*}(D)$ are equal (coalescence of equilibria). 


\section{Appendix A.4. Proof of Theorem 2}

System (11) is an asymptotically autonomous system which converges to (13). Since this last system has a finite number of hyperbolic equilibria and has no polycycles, all solutions of (11) converge to the equilibria of (13) and the set of solutions converging to $E_{2}^{2}$ is of measure 0 . This ends the proof of the theorem.

\section{Appendix A.5. Proof of Proposition 2}

The equilibria $\xi_{2}^{1}$ and $\xi_{2}^{2}$ are $\xi_{2}^{1}(D)=\left(S_{2}^{1 *}(D), \tilde{X}_{2}^{1}(D)\right)$ and $\xi_{2}^{2}(D)=$ $\left(S_{2}^{2 *}(D), \tilde{X}_{2}^{2}(D)\right)$ respectively. The distance $M_{H B}(D)=\xi_{2}^{2}(D)-\xi_{2}^{1}(D)$ is given by:

$$
\begin{aligned}
M_{H B}(D) & =\sqrt{\left(S_{2}^{2 *}(D)-S_{2}^{1 *}(D)\right)^{2}+\left(\tilde{X}_{2}^{2}(D)-\tilde{X}_{2}^{1}(D)\right)^{2}} \\
& =\sqrt{\left(S_{2}^{2 *}(D)-S_{2}^{1 *}(D)\right)^{2}+\frac{1}{k_{3}^{2} \alpha^{2}}\left(\tilde{S}_{2 i n}-S_{2}^{2 *}(D)-\tilde{S}_{2 i n}+S_{2}^{1 *}(D)\right)^{2}} .
\end{aligned}
$$

Hence

$$
M_{H B}(D)=\sqrt{1+\frac{1}{k_{3}^{2} \alpha^{2}}}\left(S_{2}^{2 *}(D)-S_{2}^{1 *}(D)\right) .
$$

\section{Appendix A.6. Proof of Proposition 3}

The equilibria $E_{2}^{1}$ and $E_{2}^{2}$ are $E_{2}^{1}(D)=\left(S_{1}^{*}, X_{1}^{*}(D), S_{2}^{1 *}(D), X_{2}^{1 *}(D)\right)$ and $E_{2}^{2}(D)=\left(S_{1}^{*}, X_{1}^{*}(D), S_{2}^{2 *}(D), X_{2}^{2 *}(D)\right)$ respectively.

The distance $M(D)=E_{2}^{2}(D)-E_{2}^{1}(D)$ is given by:

$$
\begin{aligned}
M(D) & =\sqrt{\left(S_{2}^{2 *}(D)-S_{2}^{1 *}(D)\right)^{2}+\left(X_{2}^{2 *}(D)-X_{2}^{1 *}(D)\right)^{2}} \\
& =\sqrt{\left(S_{2}^{2 *}(D)-S_{2}^{1 *}(D)\right)^{2}+\frac{1}{k_{3}^{2} \alpha^{2}}\left(S_{2 i n}^{*}(D)-S_{2}^{2 *}(D)-S_{2 i n}^{*}(D)+S_{2}^{1 *}(D)\right)^{2}} .
\end{aligned}
$$


Hence

$$
M(D)=\sqrt{1+\frac{1}{k_{3}^{2} \alpha^{2}}}\left(S_{2}^{2 *}(D)-S_{2}^{1 *}(D)\right) .
$$

\section{References}

[1] B. Benyahia, T. Sari, J. Harmand, B. Cherki. Modeling of the Soluble Microbial Products (SMP) in Anaerobic Membrane BioReactors (AMBR): Equilibria ans stability of the AM2b model. Proceeding of 18th IFAC World Congress, Milano, Italy, 2011, pp. 3789-3794.

[2] B. Benyahia, T. Sari, B. Cherki, J. Harmand. Equilibria of an anareobic wastewater treatment process and their stability. Proceeding of the 11th CAB Congress, Leuven, Belgium, 2010, pp. 371-376.

[3] G. Lyberatos and I.V. Skiadas. Modelling of Anaerobic Digestion - A Review. Global Nest: the Int. J. 2 (1999) 63-76.

[4] H.L. Smith and P. Waltman. The theory of the chemostat: Dynamics of microbial competition. Cambridge University Press, 1995.

[5] H.R. Thieme. Asymptotically Autonomous Differential Equations in the Plane. Rocky Mountain J. Math. 24 (1) (1993) 351-380.

[6] I. Simeonov and S. Diop. Stability analysis of some nonlinear anaerobic digestion models. Int. J. BIOautomation 14 (1) (2010) 37-48.

[7] IWA Task Group for Mathematical Modeling of Anaerobic Digestion Processes. Anaerobic digestion model no. 1 (adm1). Technical report, IWA (International Water Association), 2001. 
[8] J. Hess, and O. Bernard. Advanced Dynamical Risk Analysis for Monitoring Anaerobic Digestion Process. AIChE 25 (3) (2009) 643-653.

[9] J. Hess, and O. Bernard. Design and Study of a risk management criterion for an unstable anaerobic wastewater treatment process. J. Process Control 18 (2008) 71-79.

[10] M. Sbarciog, M. Loccufier, E. Noldus. The estimation of stability boundaries for an anaerobic digestion system. Proceeding of the 11th CAB Congress, Leuven, Belgium, 2010, pp. 359-363.

[11] N. Dimitrova and M. Krastanov. Nonlinear stabilizing control of an uncertain bioprocess model. Int. J. Appl. Math. Comput. Sci., 19 (3) (2009) 441-454.

[12] O. Bernard, Z. Hadj-Sadock, D. Dochain, A. Genovesi, and J.-P. Steyer. Dynamical Model Development and Parameter Identification for an Anaerobic Wastewater Treatment Process. Biotechnology and Bioengineering, 75 (2001) 424-438.

[13] P. Le-Clech, V. Chen, and T.A.G. Fane. Fouling in membrane bioreactors used in wastewater treatment. Journal of Membrane Science, 284 (2006) 17-53.

[14] S. Shen, G. C. Premier, A. Guwy and R. Dinsdale. Bifurcation and stability analysis of an anaerobic digestion model. Nonlinear Dyn., 48 (2007) 391-408.

[15] S. Marsili-Libelli and S. Beni. Shock load modelling in the anaerobic digestion process. Ecol. Model., 84 (1996) 215-232. 